\title{
Simulating, Planning and Analysing Multiple-Pointing VLBI Surveys
}

\section{John Morgan}

International Centre for Radio Astronomy Research, Curtin University, Perth, Australia

E-mail: john.morgan@icrar.org

In recent years, advances in correlation and data reduction techniques have opened up the entire primary beam to VLBI study. Already observations have been made where sources across the primary beam have been detected and imaged [2]. The next step is to move to multiple pointings. In this poster I will generalise the widely-used formula for calculating the image thermal noise limit to give the sensitivity across the primary beam. I will then present simulations of the sensitivity across the wide field for different VLBI networks, both homogeneous and heterogeneous, exploring the use of multiple pointings. I will then comment on the impact this has on calibration strategies.

10th European VLBI Network Symposium and EVN Users Meeting: VLBI and the new generation of radio arrays

September 20-24, 2010

Manchester Uk 


\section{Introduction}

Imaging to the edge of the primary beam is routinely done for connected-element arrays. In this case a correction is applied to the intensity across the final image. However for heterogeneous arrays it is more appropriate to apply an antenna-by-antenna correction. This is achievable if we assume that the primary beam response is constant across each image. The primary beam power function of a baseline is given by

$$
A_{12}(l, m)=F_{1}^{*}(l, m) \times F_{2}(l, m),
$$

where $F_{1}$ and $F_{2}$ are the voltage patterns of the two antennas in the $l, m$ plane [1]. The primary beam corrected amplitude of each visibility $I_{i j r}$ on the baseline $i, j$ in a dataset phase-centred in the $l, m$ plane can be calculated from the observed amplitude $I_{o b s}$ :

$$
I_{i j}(l, m)=\frac{I_{o b s}}{A_{i j}(l, m)}
$$

where the amplitude of the power function has been scaled to 1 at the centre of the primary beam. It is also possible to calculate the theoretical RMS noise limit:

$$
\Delta I_{m}=\frac{1}{\eta_{s} \cdot \sqrt{2 \cdot \Delta v \cdot \tau_{i n t}}} \times\left(\sum_{i=1, j=i+1}^{i=N-1, j=N} \frac{\left|A_{i, j}(l, m)\right|^{2}}{S E F D_{i} \times S E F D_{j}}\right)^{-1 / 2}
$$

where the sum is over all baselines. It is trivial to extend this to multiple pointings (by simply considering them as different baselines with different primary beam power functions). It should be clear from equation (1.1) that the primary beam response is maximised if both antennas point at the same place.

\section{Simulations}

The primary beam has been modelled using a radial sinc function truncated at the first null and the RMS sensitivity has been calculated across the primary beam in fig. 1 (assuming uniform weighting) for the VLBA and various multiple-pointing configurations of the EVN. The EVN has a far larger collecting area than the VLBA, however the sensitivity is concentrated in the baselines to the larger antennas. This results in a highly-sensitive area at the centre corresponding to the primary beam of the larger antennas (fig. 2). In the multiple-pointing observations the Effelsberg primary beam is Nyquist sampled and the other primary beams overlap. This broadens the central, high-sensitivity beam centre. The baselines composed of two smaller antennas contribute a limited amount of sensitivity far from the beam centre which may be useful for finding potential calibrator sources for future studies. Figure 4 shows the importance of using the contribution of the larger antennas beyond their Half-Power Beam Width (HPBW).

These simulations have already proved useful in planning wide-field VLBI observations (fig. 3). It is intended to expand this software to assist in the correlation and calibration of wide-field data. This will require further knowledge of the array geometry to account properly for non-radial primary beams. 


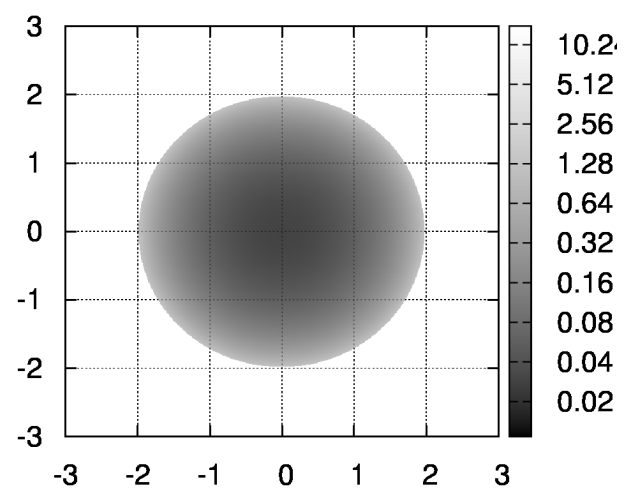

(a) VLBA 1 pointing

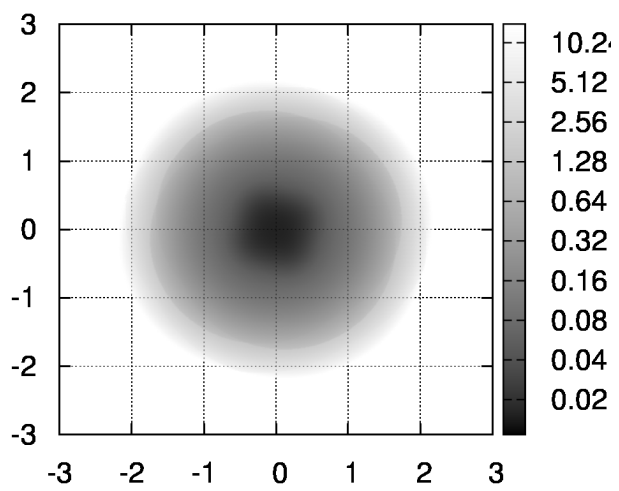

(c) EVN 4 pointings

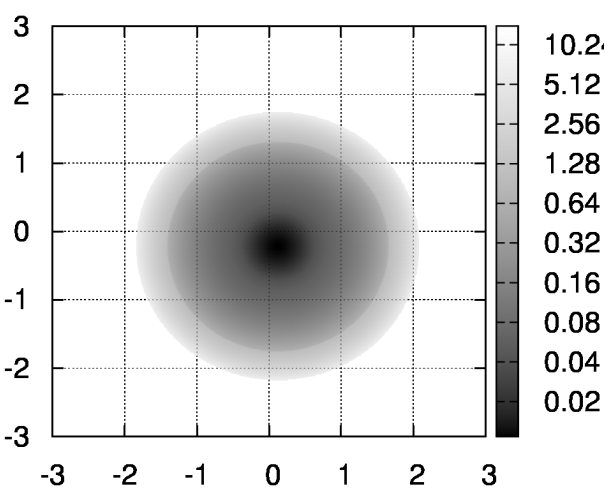

(b) EVN 1 pointing

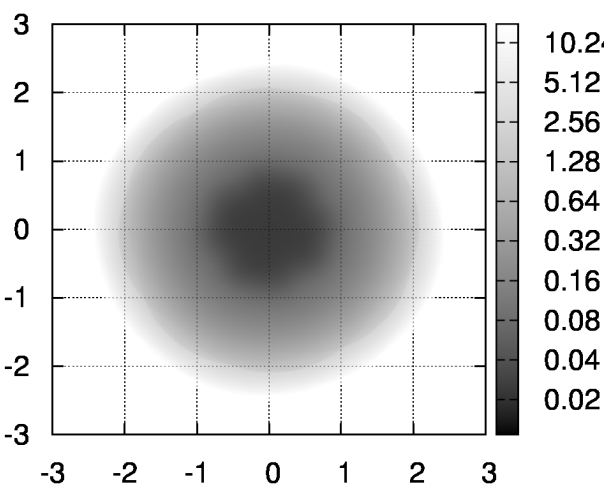

(d) EVN 12 pointings

Figure 1: Predictions of the RMS thermal noise for approx 5 hours of observing time and a recording rate per antenna of $512 \mathrm{Mbps}$. Scale in mJy; $l$ and $m$ scaled to the Half Width Half Maximum (HWHM) of the VLBA.

\section{Acknowledgements}

The author would like to thank Megan Argo for preparing fig. 3.

\section{References}

[1] [1] R. Strom: What is the primary beam response of an interferometer with unequal elements? European VLBI Network on New Developments in VLBI Science and Technology, 2004.

[2] [2] E. Middelberg et al.: Wide-field VLBA Observations of the Chandra Deep Field South. Accepted: Astronomy and Astrophysics 


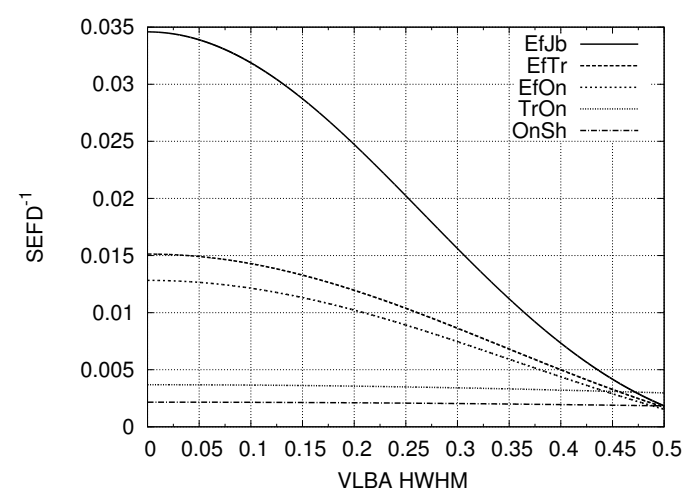

Figure 2: A measure of the sensitivity of each baseline taking both the primary beam and the SEFD of each antenna into account

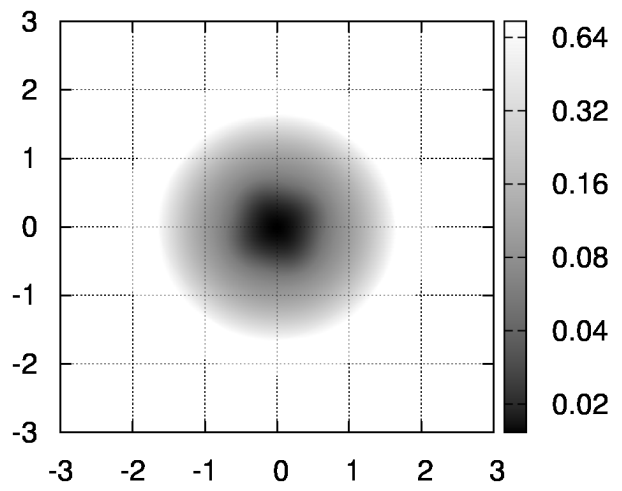

(a) Truncating at $180 \%$ of the HPBW

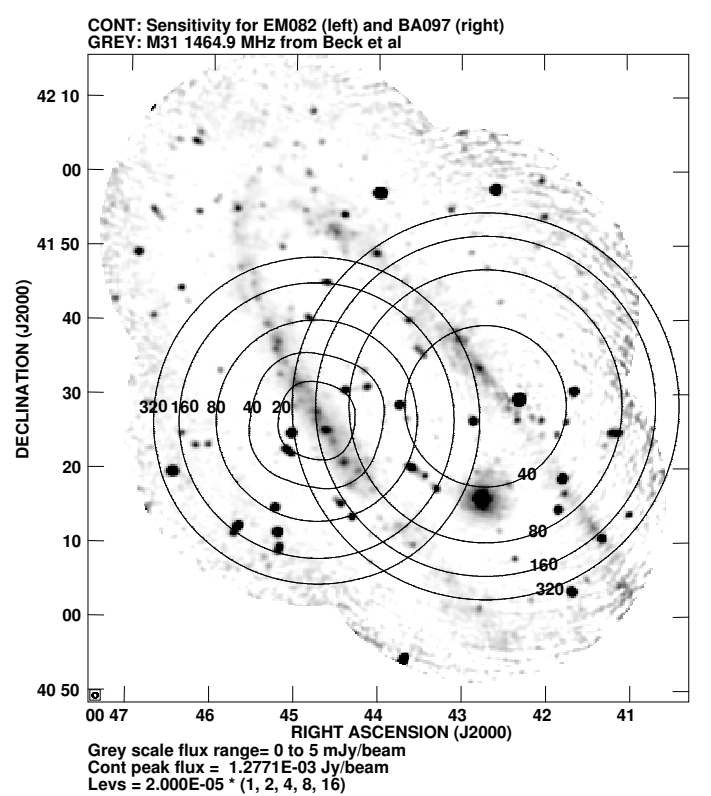

Figure 3: EVN and VLBA pointing sensitivity predictions plotted together in AIPS with VLA data in background. Contour units are in $\mu \mathrm{Jy}$

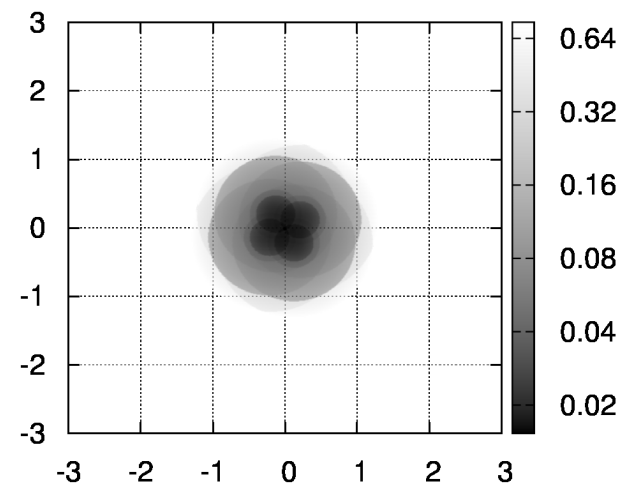

(b) Truncating at $100 \%$ of the HPBW

Figure 4: Predicted rms noise level across the primary beam truncating each baseline at either $100 \%$ of $180 \%$ of the HPBW of the larger antenna on the baseline. Each antenna primary beam is modeled as a sinc function. 